\title{
Synthesis and Biological activity of 1,3-Thiazolylidenehydrazinylidene ethylpyridiniumbromide monohydrate, 1,3- \\ Thiazolylidenehydraziniumbromide and 1,3-Thiazolylidenehydrazine derivatives
}

\author{
Alaa A. Hassan ${ }^{a}$, Shaaban K. Mohamed ${ }^{\star}$, Nasr K. Mohamed ${ }^{a, b *}$, Kamal M. A. El-Shaieb ${ }^{a}$, \\ Ahmed T. Abdel-Aziz a and Marwa Rageh Abdel Rahman ${ }^{c}$ \\ ${ }^{a}$ Chemistry Department, Faculty of Science, Minia University, El-Minia 61519, Egypt. \\ ${ }^{b}$ Chemistry and Environment Division, Manchester Metropolitan University, Manchester, MI5GD. England. \\ shaabankamel@yahoo.com \\ ${ }^{c}$ Botany Department, Faculty of Science, Sohag University, 82524 Sohag, Egypt
}

\begin{abstract}
1,3-Thiazolylidenehydrazinylidene ethylpyridinium bromide monohydrate, 1,3-thiazolylidenehydrazinium bromide and 1,3thiazolylidenehydrazine derivatives were synthesized by heterocyclization of 2-(1-substituted ethylidene)hydrazinecarbothioamides, characterized and screened for their anti-bacterial activities. $3 \mathbf{h}$ showed the highest inhibitory effect against all types of bacterial compared to Moxifloxacin. The structures of synthesized compounds were established by spectroscopic (IR, ${ }^{1} \mathrm{H},{ }^{13} \mathrm{C}-\mathrm{NMR}$, Mass) and X-ray analyses.
\end{abstract}

\section{Keywords}

Substituted ethylidenehydrazinecarbothioamides, 1-aryl-2-bromoethanones, 1,3-thiazolylidene-hydrazine derivatives and their salts, anti-bacterial activity.

\section{Academic Discipline And Sub-Disciplines}

Chemistry

\section{SUBJECT CLASSIFICATION}

Organic Synthesis

\section{TYPE (METHOD/APPROACH)}

Cycloaddition elimination reactions

\section{Council for Innovative Research}

\section{Peer Review Research Publishing System}

Journal: Journal of Advances in Chemistry

Vol. 11, No. 3

editorjaconline@gmail.com 


\section{INTRODUCTION}

Compounds possessing Schiff bases are well known for their phamacological properties as antifungal, anti-cancer and anti-viral agents $[1,2]$.

Thiosemicarbazones reacted with cyclization reagents such as dichloroacetone, ethylchloroacetate, ethyl-1,2chloroacetoacetate and 2-bromoacetophenone to give substituted (benzylidenehydrazono)thiazoles [3-6].

A NBS-mediated sequential one-pot synthes is of multi-functionalized thiazoles from 1,3-dicarbonyl compounds and mercaptonitrile salts has been developed under mild conditions [7].

The occurrence of thiazole ring system in numerous biologically active molecules have recognized and possess wide ranges of biological properties such as antioxidant [8], antibacterial drugs [9-11], fungicidal treatment [3,12], antiinflammatory [13-16], anticonvulsant [17], and anti-HIV [18-20]. 2-Aminothiazole was used as inhibitors of human cancer and Alzheimer's dis ease [21-24].

The most frequently used synthetic method of generating thiazoles is the Hantzsch process [25], in which $\alpha$-haloketone is condensed with thioamides. Several methods for the synthesis of tri-substituted thiazoles have been reported [26-29].

$\mathrm{N}$-substituted pyridinium salts are widly used in organic synthesis [30-32], and have much attention in the field of nonlinear optics (NLO) $[30,31,33]$ as well as antibacterial and antifungal activities [34].

In view of these, it was thought of interest to combine both pharmacophoric moieties 1,3-thiazole and aromatic compounds (with donating and withdrawing groups) as well as heterocyclic ring (such as pyridine).

A series of substituted 1,3-thiazole derivatives were synthesized and evaluated for their antibacterial activity.

\section{RESULTS AND DISCUSSION}

Our investigation was performed by reacting 2-(1-substituted-ethylidenehydrazinecarbothioamides 1a-h with 1-aryl-2bromoethanones $\mathbf{2 a , b}$ in refluxing ethanol/ piperidine, the general synthetic pathway was depicted in $($ Scheme 1$)$. [ $[3,4$ Diaryl-1,3-thiazolylidene)(hydrazineylid-ene)]ethyl-pyridinium bromide monohydrates (3a,b), (3,4-diaryl-1,3thiazolylidene)ethylidenehydrazinium bromides (3c,d) and (3,4-diaryl-1,3-thiazolylidene)hydrazines (3e-h) were obtained in (80-89\%) yields as three different types of thiazole products from the reaction of $\mathbf{1 a - h}$ with $\mathbf{2 a , b}$.

A detailed interpretation of IR bands of $\mathbf{3 a}$ is discussed. The IR spectrum of $\mathbf{3 a}$ shows a broad band at $3361 \mathrm{~cm}^{-1}$ which can be attributed to NH. The (N-N) stretching band in thiazole derivative $3 a$ observed at $1567 \mathrm{~cm}^{-1}$. The IR of $3 a$ revealed strong band at $1619 \mathrm{~cm}^{-1}$ due to $(C=N)$, the $(C-S-C)$ band was observed at $767 \mathrm{~cm}^{-1}$ [6]. A broad band at 3448 due to the $\mathrm{OH}$ of $\mathrm{H}_{2} \mathrm{O}$ molecule.

The ${ }^{1} \mathrm{H}$ NMR of $3 \mathrm{a}$ chosen as prototype showed that the pyridine-nitrogen still had a proton and pyr- $\mathrm{NH}$ resonance at 9.17 ppm, four proton multiplets at $8.20-8.28$ due to pyridine- $\mathrm{CH}$, two singlets at 2.32 and 6.42 because the proton of $\mathrm{CH}_{3}$ and thiazole- $\mathrm{CH}$, respectively. A broad signal at $1.6 \mathrm{ppm}$ due to $\mathrm{OH}$ of one molecule of $\mathrm{H}_{2} \mathrm{O}$. Multiplets at 7.12-7.78 accounting for ten aromatic protons.

The ${ }^{13} \mathrm{C}$ NMR spectrum of 3 a supported the ${ }^{1} \mathrm{H}$ NMR spectroscopic data by the distinctive appearance of carbon signals representing acyclic $\mathrm{C}=\mathrm{N}$ at $\delta \mathrm{H}=157.22 \mathrm{ppm}$, thiazole $\mathrm{C} 2, \mathrm{C} 4$ resonate at 168.12 and $148.55 \mathrm{ppm}$, respectively and thiazole- $\mathrm{CH}$ at 104.06. Furthemore, in the ${ }^{13} \mathrm{C}$ NMR of $\mathbf{3 a}, \mathbf{b}$, the pyridine-C2 is downfield shifted 148.55-147.12. The mass spectrometry fragmentation of $3 a$ was studied under electron ionization. Loss of $\left(\mathrm{HBr}+\mathrm{H}_{2} \mathrm{O}\right)$ giving rise to the ion $\mathrm{m} / \mathrm{z}=363$. The resulting fragment ions undergo loss of 104 a.m.u. $\left[\mathrm{C}_{6} \mathrm{H}_{5}-\mathrm{C}\left(\mathrm{CH}_{3}\right)\right]$, and 135 a.m.u. $(\mathrm{Ph}-\mathrm{N}=\mathrm{C}=\mathrm{S})$. 


\section{Scheme 1}

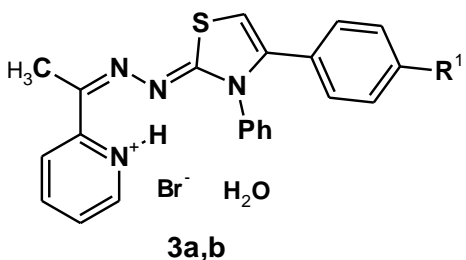<smiles>C1CCCCC1</smiles><smiles>[R]/C(C)=N/NC(=S)Nc1ccccc1</smiles><smiles>O=C(CBr)c1ccc(P)cc1</smiles>

1a-h

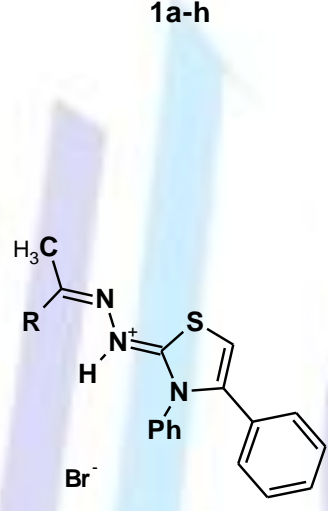

$3 c$<smiles>[R]C(C)=N/N=c1\scc(-c2ccc(Br)cc2)n1-c1ccccc1</smiles>

3d

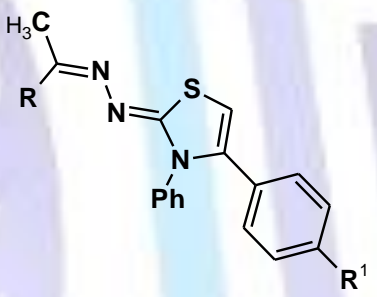

$3 e-h$

\begin{tabular}{|l|l|l|l|l|l|l|l|l|}
\hline $\begin{array}{l}\text { 1a-h } \\
\text { and } \\
\text { 3a-h }\end{array}$ & $\mathbf{a}$ & $\mathbf{b}$ & $\mathbf{c}$ & $\mathbf{d}$ & $\mathbf{e}$ & $\mathbf{f}$ & $\mathbf{g}$ & $\mathbf{h}$ \\
\hline $\mathrm{R}$ & & & & & & & & \\
\hline $\mathrm{R}^{1}$ & $\mathrm{H}$ & $\mathrm{Br}$ & $\mathrm{H}$ & $\mathrm{Br}$ & $\mathrm{H}$ & $\mathrm{Br}$ & $\mathrm{H}$ & $\mathrm{Br}$ \\
\hline
\end{tabular}

The molecular structure of $\mathbf{3 a}$ was established by single crystal X-ray analysis (Fig. 1) [35]. The dihedral angle between $\mathrm{S} 1 / \mathrm{N} 1 \mathrm{C} 1-\mathrm{C} 3$ thiazolylidene and N4/C18-C22 pyridinium rings is $14.73 \mathrm{C}(3) \mathrm{o}$ while that between the phenyl groups $\mathrm{C} 4-\mathrm{C} 9$ and C10-C15 and the mean plane of the thiazolylidene ring are $34.69(13)$ and $64.27(13)^{0}$, respectively. 
Fig. 1. Molecular conformation of $3 a$ with $50 \%$ probability displacement eliposids

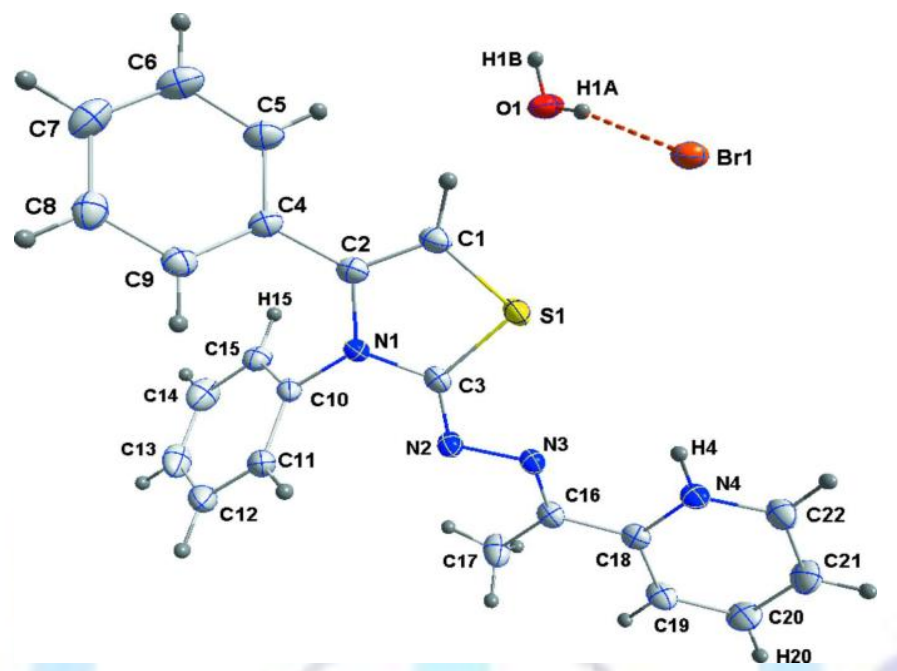

In the crystal zigzag chains of altemating bromide ions and water molecules associated through $\mathrm{O}-\mathrm{H}$......Br interactions run in channels approximately parallel to the $b$ axis [35].

On the other hand, 2-((1E)-1-\{2-[(2E)-4-(4-bromo-phenyl)-3-phenyl-2,3-dihydro-1,3-thiazol-2-ylidene]hydr-azine-1ylidene\}ethyl)-pyridinium bromide monohydrate formed during the reaction of $\mathbf{1} \mathbf{b}$ with $\mathbf{2} \mathbf{b}$. In the crystal structure of the title hydrated salt $\mathrm{C}_{22} \mathrm{H}_{18} \mathrm{BrN}_{4} \mathrm{~S}+\mathrm{Br}-. \mathrm{H}_{2} \mathrm{O}$ the $\mathrm{N} 4 / \mathrm{C} 18-\mathrm{C} 22, \mathrm{C} 1-\mathrm{C} 6$ and $\mathrm{C} 10-\mathrm{C} 15$ aromatic rings make dihedral angles of 14.20 (12), 34.29 (10) and $68.75(11)^{0}$, respectively with the (S1/N1/C7-C9) thiazole ring (Fig. 2) [36].

Fig. 2. The crystal structure of the hydrated salt $\mathrm{C}_{22} \mathrm{H}_{18} \mathrm{BrN}_{4} \mathrm{~S}+. \mathrm{Br}-. \mathrm{H}_{2} \mathrm{O}(3 \mathrm{~b})$.

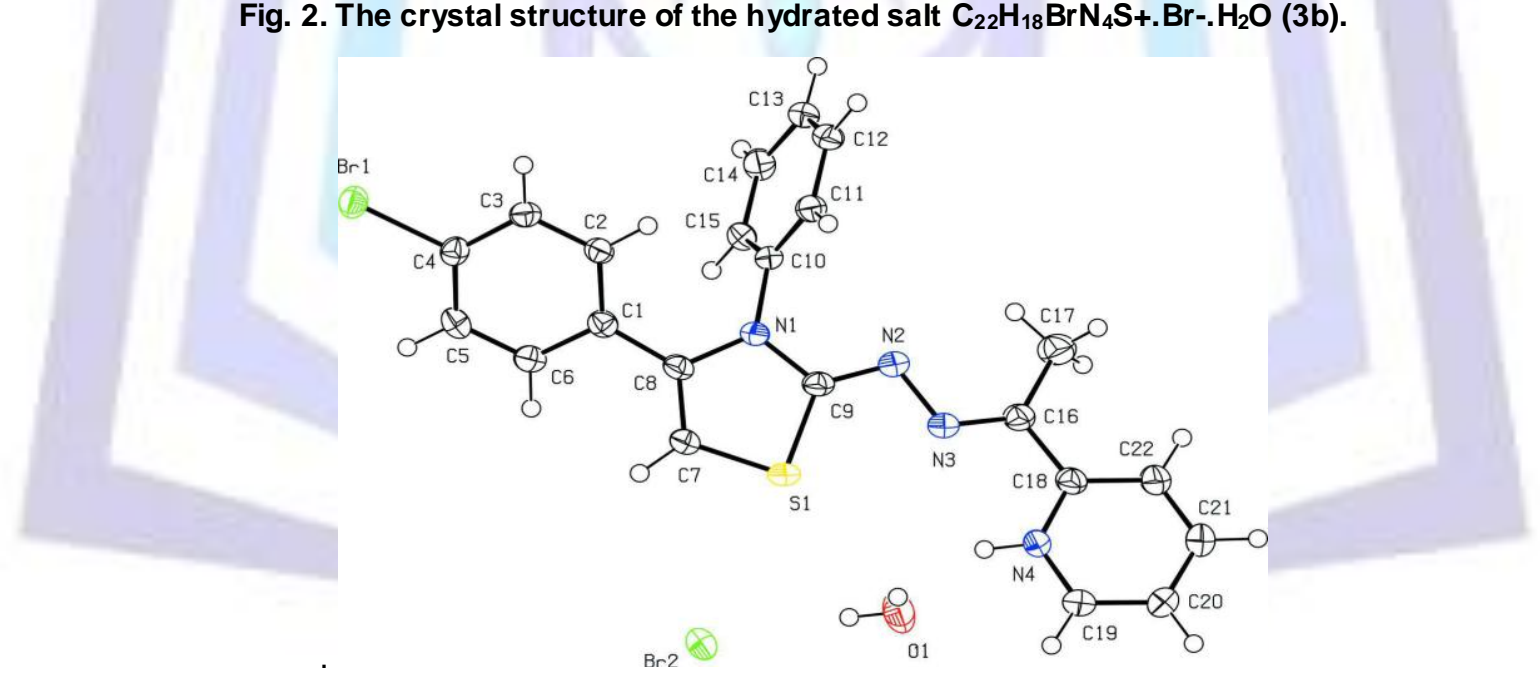

The elemental analyses and mass spectra of $\mathbf{3 c}, \mathbf{d}$ clearly revealed that the products were formed by the addition of one molecule of $\mathbf{1 c}, \mathbf{d}$ to one molecule of $\mathbf{2} \mathbf{a}, \mathbf{b}$ with elimination one molecule of $\mathrm{H}_{2} \mathrm{O}$. The IR spectrum of $\mathbf{3 c}$ showed absorption of phenolic- $\mathrm{OH}$ and $\mathrm{NH}$ at 3423 and $3332 \mathrm{~cm}^{-1}$, two strong bands at 1612,1564 due to $\mathrm{C}=\mathrm{N}$ vibrations and $(\mathrm{N}-\mathrm{N})$ stretching, respectively.

The ${ }^{1} \mathrm{H}$ NMR spectrum of 3c, two broad signals at 9.51 and 9.20 ppm due to phenolic-OH, and hydrazinium-NH respectively. In the ${ }^{13} \mathrm{C}$ NMR spectrum of $3 c$ signals at $\delta C=157.10,168.53$ and $148.79 \mathrm{ppm}$ were assigned to acyclic $\mathrm{C}=\mathrm{N}$, thiazole-C2 and thiazole-C4, respectively. The following additional remarks are necessary: The ${ }^{1} \mathrm{H}$ NMR spectrum of $3 \mathrm{c}$ shows the presence of one singlet at $\delta \mathrm{H}=2.31 \mathrm{ppm}$ due to $\mathrm{CH}_{3}$, multiplets at $6.84-7.77$ due to aromatic protons. The ${ }^{13} \mathrm{C}$ NMR shows the $\mathrm{CH}_{3}$ group at 14.15 and thiazole- $\mathrm{CH}$ at $102.83 \mathrm{ppm}$.

The single crystal X-ray structure analysis of (Z)-1-[(2E)-3,4-diphenyl-2,3-dihdro-1,3-thiazol-2-ylidene]-2-[1-(4hydroxyphenyl)ethylidene]hydrazinium bromide (3c) confimed that, the dihydrothiazole ring [r.m.s. deviation= $0.015 \AA$ ) is twisted with respect to each of $\mathrm{C}$ - and $\mathrm{N}$-bound phenyl rings and the hydroxy benzene ring, making dihedral angles of 76.0 
(2), 71.2 (2) and $9.8(2)^{0}$, respectively (Fig. 3) [37]. In the crystal inversion related molecules are linked by association of the bromide ions with the cations via $\mathrm{N}-\mathrm{H}$....Br and $\mathrm{O}-\mathrm{H}$....Br hydrogen-bonding interactions [37].

Fig. 3. Single crystal $x$-ray structure of $3 c$

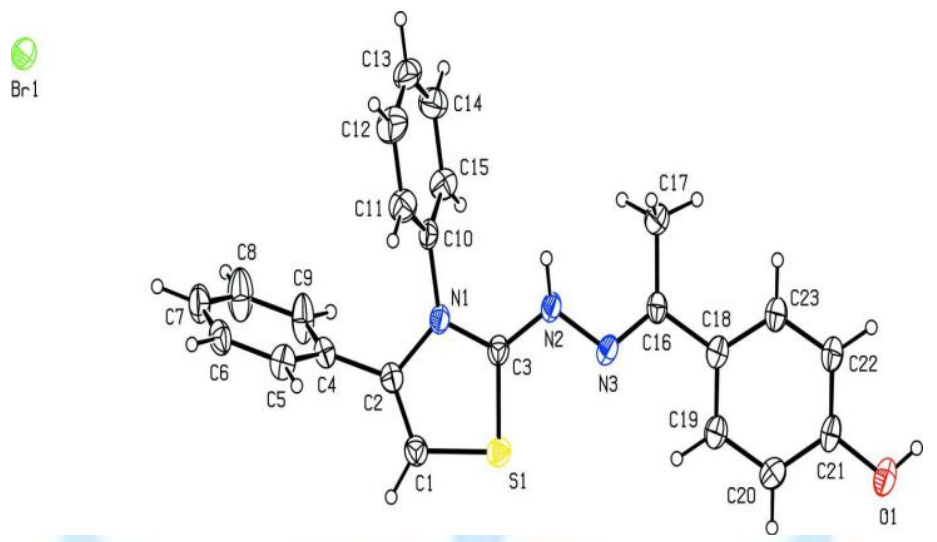

Substitution the $p$-hydrogen atom of benzene ring attached to thiazole-C4 by bromide afforded the title compound $\mathbf{3 d}$. For further elucidation the structure of $\mathbf{3 d}$, the single crystal X-ray diffraction has carried out and showed that the bromophenyl, phenyl and phenol rings make dihedral angles of 46.5 (1), 66.78 (8) and 15.4 (2), respectively, with the mean squares plane of the thiazolidene ring. In the crystal, the lattice water molecule is hydrogen bonded to the phenol group and makes a weaker $\mathrm{O}-\mathrm{H} \cdots \mathrm{N}$ connection (Fig. 4) [38].

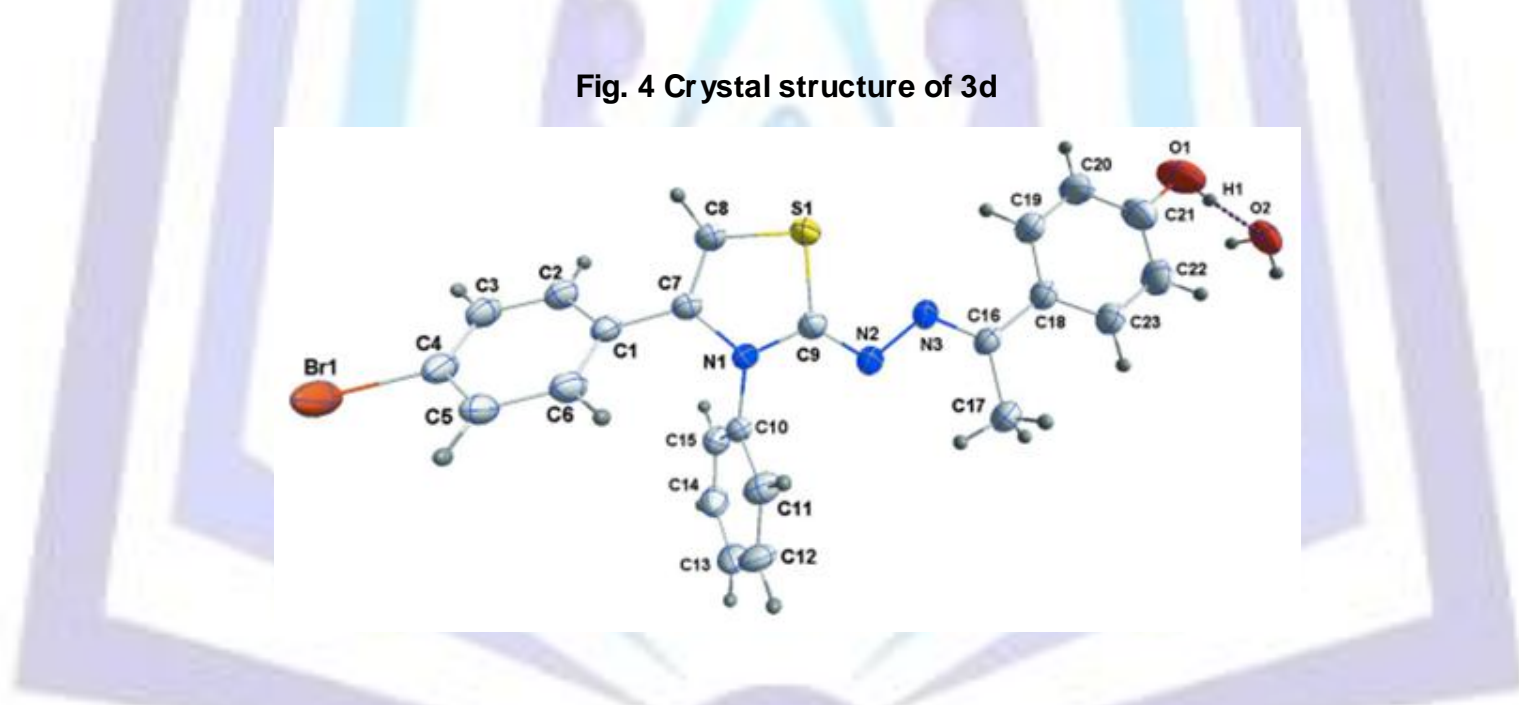

The structures of the third type of the products containing compounds $3 \mathbf{e}-\mathbf{h}$ were assigned using spectroscopic tools such as IR, NMR $\left({ }^{1} \mathrm{H}-,{ }^{13} \mathrm{C}\right)$ and mass spectrometry as well as single crystal $\mathrm{X}$-ray analyses.

The mass spectrometry and elemental analyses confirm the molecular masses and molecular composition of $3 \mathbf{e}-\mathbf{h}$, which obtained from reacting one molecule of $\mathbf{1 e - h}$ and one molecule of $\mathbf{2} \mathbf{a}, \mathbf{b}$ via loss a molecule of $\mathrm{H}_{2} \mathrm{O}$ and another of $\mathrm{HBr}$.

The ${ }^{1} \mathrm{H}$ NMR spectra of $3 \mathrm{e}-\mathrm{h}$ showed the presence of two singlets with the ratio $3: 1$ at 2.27-2.38 and 6.17-6.21 to $\mathrm{CH}_{3}$ and thiazole- $\mathrm{CH}$, respectively, in addition to aromatic protons. The ${ }^{1} \mathrm{H}$ NMR of $3 \mathbf{e}, \mathbf{f}$ clearly indicated the presence of extra $\mathrm{CH}_{3}$ centered at $2.35-2.58 \mathrm{ppm}$. The presence of thiazole- $\mathrm{CH}$ was also proved by ${ }^{13} \mathrm{C}$-DEPT NMR spectrum, exhibiting positive signals at 101.72-103.14 ppm. Signals at 168.51-169.67 and 148.86-149.12 were observed due to thiazole-C2 and thiazole-C4.

In (1Z,2E)-1-(3,4-diphenyl-2,3-dihydro-1,3-thiazol-2-ylidene)-2-(1-p-tolylrthylidene)hydrazine (3e), the thiazole ring makes dihedral angles of $52.03(6), 62.63(6)$ and $12.35(6)^{0}$, respectively, with two phenyl rings and the benzene ring (Fig. 5) [39]. 
Fig. 5. Molecular structure of $3 e$

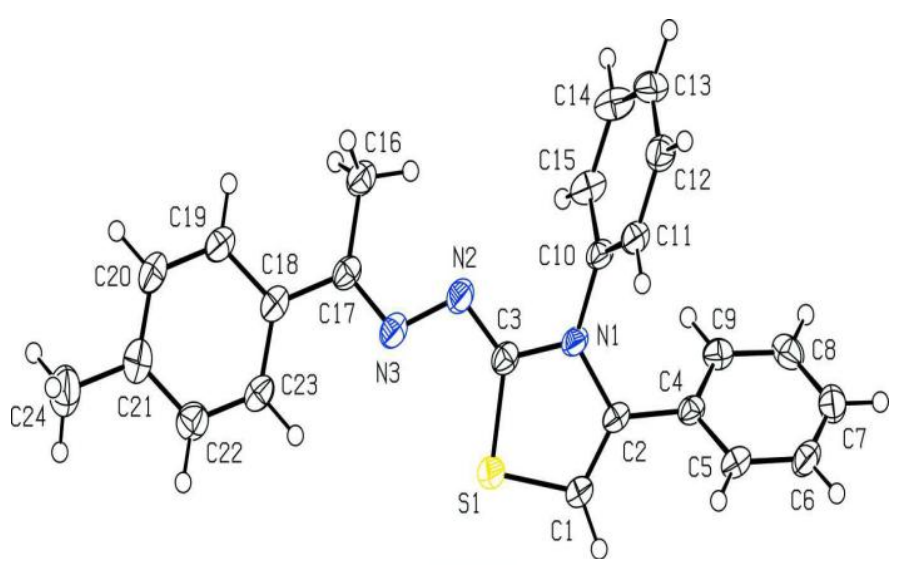

Moreover, in (2E)-4-(4-bromophenyl)-2-((2Z)-(1-(4-methy(phenyl)ethylidene)hydrazinylidene)-3-phenyl-2,3-dihydro-1,3thiazole (3f), the dihydrothiazele ring is approximately plannar with a maximum deviation of 0.08 (2) $\AA$, and is twisted with respect to the 4-bromophenyl ring, the phenyl ring and methyl phenyl ring, making dihedral angles of 47.96 (8), 59.52 (9) and $16.96(9)^{0}$, respectively (Fig. 6) [40].

Fig. 6. Perspective view of $3 f$ showing the planarity of thiazole ring

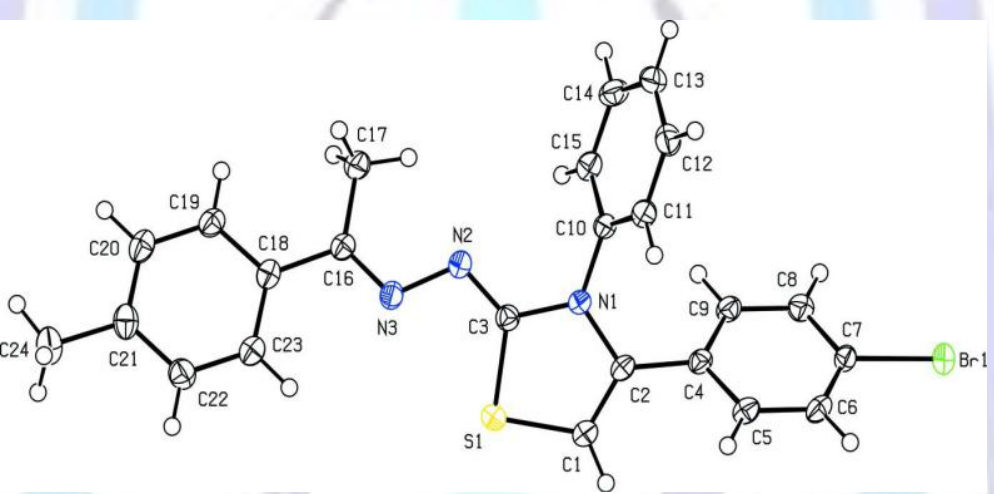

A rational for the formation of compounds $\mathbf{3 a - h}$ as depicted in (Scheme 2) via the formation of intermediate $\mathbf{4 a - h}$ followed by elimination a molecule of $\mathrm{HBr}$ and another of $\mathrm{H}_{2} \mathrm{O}$.

\section{Scheme 2}

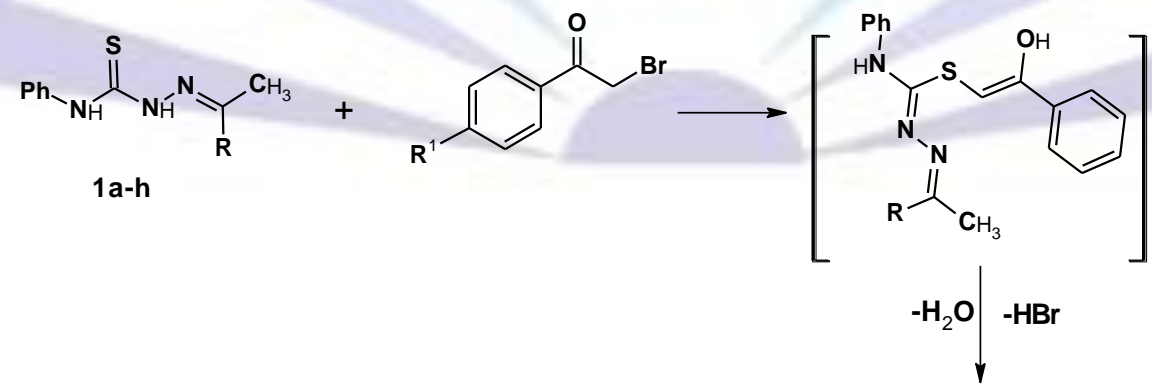

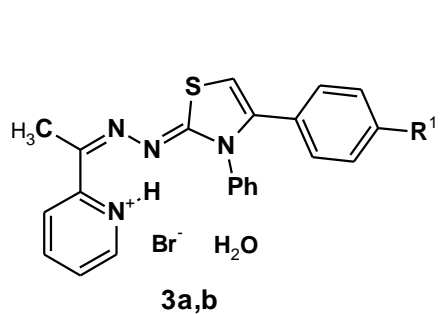

$3 a, b$<smiles>[R]C(C)=N/N=c1\scc(-c2ccccc2)n1-c1ccccc1</smiles>

$3 c$<smiles>[R]C(C)=NN=c1scc(-c2ccc(Br)cc2)n1-c1ccccc1</smiles>

3d<smiles>[R]C(C)=NN=c1scc(-c2ccc([R])cc2)n1-c1ccccc1</smiles>

3e-h 


\section{Conclusion}

In summary, we have described the synthesis for four types of thiazolylidenhydrazine derivatives via heterocyclization of 2-(1-substituted ethylidene)hydrazinecarbothio-amides by the reaction with 1-aryl-2-bromoethanones. The first type of products is substituted 1,3-thiazolylidene-hydrazonylidene ethylpyridinium bromide monohydrate, whereas substituted 1,3thiazolylidenehydrazinium bromide is the second type of products. The third, is the hemihydrate of 1,3-thiazolylidene hydrazine derivatives in addition to the derivatives of 1,3-thiazolylidene hydrazine as the fourth type. Among all types of 1,3-thiazolylidene hydrazines, 3h showed the highest inhibitory effect against all gram positive and gram negative bacterial strains compared to the standard broad spectrum antibiotic Moxifloxacin.

\section{Experimental}

Melting points were determined using open glass capillaries on a Gallenkamp melting point apparatus (Weiss Gallenkamp, loughborough, UK) and are uncorrected. Infrared spectra $\left(\mathrm{U} / \mathrm{cm}^{-1}\right)$ were recorded from potassium bromide disks with a Shimadzu 408 (Shimadzu corporation, Kyoto, Japan). ${ }^{1} \mathrm{H}$ - and ${ }^{13} \mathrm{C}-\mathrm{NMR}$ spectra $\left(400 \mathrm{MHz}\right.$ for ${ }^{1} \mathrm{H}, 100 \mathrm{MHz}$ for ${ }^{13} \mathrm{C}$ ) were observed in $\mathrm{CDCl}_{3}$ on DELTA2-NMR spectrometer (DELTA2, Manchester Metropolitan University, United Kingdom) with tetramethylsilane as the internal standard. The ${ }^{13} \mathrm{C}-\mathrm{NMR}$ signals were assigned with the aid of DEPT $135 / 90$ experiments. Mass spectra (70 eV, electron impact mode) were recorded on Finnegan MAT 312 (Germany) instrument. X-ray diffractions were measured using Bruker SMART APEX CCD diffractometer with Graphite monochromator and fine-focus scaled tube as a Radiation source. Thin layer chromatography (TLC) was performed on analytical Merck 9385 Silica aluminium sheets (Kieselgel 60) with Pf 254 indicator, TLC's were viewed at $\lambda \mathrm{max}=254 \mathrm{~nm}$. Elemental analyses were carried out at Microanalytical Center, Cairo University, Egypt.

\section{Starting Materials}

2-(1-Substituted ethylidene)hydrazinecarbothioamides 1a-h were prepared by condensation 4-phenylthiosemicarbazide with the appropriate ketones according to the published procedures [41,42]. 1-Aryl-2-bromoethanones (2a,b) were prepared according to Nobuta and Salama $[43,44]$. Anti-bacterial activity has been carried out at Botany Department, Sohag University.

\section{Products}

\section{Synthesis of 1,3-thiazolylidenehydrazinylidene pyridinium salts and 1,3- thiazolylidenehydrazine derivatives 3a-h}

\section{General procedure}

A mixture of (1 mmol) 2-(1-substituted ethylidene)hydrazine-carbothioamides $\mathbf{1 a - h}$ and (1 mmol) of 1-aryl-2brom oethanones $(2 \mathbf{a}, \mathbf{h})$ in absolute ethanol $(30 \mathrm{ml})$ was refluxed for $\mathbf{6 - 8} \mathbf{h}$, then cooled to room temperature. A yellow solid precipitated, it was filtered and washed with a small amount of ethanol and recrystallized from ethanol to afford good quality crystals.

\section{2-((1E)-1-\{2-[(2Z)-3,4-Diphenyl-2,3-dihydro-1,3-thizol-2-ylidene]hydrazine-1-ylide ne \}ethyl)pyridin-1-ium} bromide monohydrate (3a). Yellow crystals (ethanol), m.p $234-236{ }^{\circ} \mathrm{C}(0.38 \mathrm{~g}, 81 \%), \quad \mathrm{IR}(\mathrm{KBr})=3361\left(\mathrm{Pyr}-{ }^{+} \mathrm{NH}\right), 1619$ $(\mathrm{C}=\mathrm{N}), 1567(\mathrm{~N}-\mathrm{N})$ stretching. ${ }^{1} \mathrm{H}-\mathrm{NMR}\left(\mathrm{CDCl}_{3}\right) \delta_{\mathrm{H}}=1.61\left(\mathrm{br}, 2 \mathrm{H}, \mathrm{H}_{2} \mathrm{O}\right), 2.32\left(\mathrm{~s}, 3 \mathrm{H}, \mathrm{CH}_{3}\right), 6.42(\mathrm{~s}, 1 \mathrm{H}$, thiazole-CH), 7.12-7.23 (m, 2H, Ar-H), 7.24-7.26 (m, 4H, Ar-H), 7.28-7.33 (m, 4H, Ar-H), 8.20-8.28 (m, 4H, pyr-H), $9.17(b r, 1 H, p y r-$ $\left.{ }^{+} \mathrm{NH}\right) .{ }^{13} \mathrm{C} \mathrm{NMR}\left(\mathrm{CDCl}_{3}\right) \delta_{\mathrm{C}}=14.17\left(\mathrm{CH}_{3}\right), 104.06$ (thiazole-CH), 123.24, 123.51, 128.26, 128.39, 128.50, $128.94(\mathrm{Ar}-\mathrm{CH})$, 130.41, 130.80 (pyridine-CH), 131.05, 137.08 (Ar-C), 141.20, 141.63 (pyridine-CH), 146.37 (pyridine-C), 148.55 (thiazoleC4), 157.22 (acyclic C=N), 168.12 (thiazole-C2). Ms. m/z (\%) $363\left(\mathrm{M}^{+}-\left(\mathrm{HBr}+\mathrm{H}_{2} \mathrm{O}\right), 23\right), 263(10), 236(18), 135(27), 104$ (76), 77 (100). Anal. Calod for $\mathrm{C}_{22} \mathrm{H}_{21} \mathrm{BrN}_{4} \mathrm{O}_{5}$ (469.40), C, 56.29; H, 4.51; Br, 17.02; N, 11.94; S, 6.83. Found C, 56.17; H, $4.61 ; \mathrm{Br}, 16.88 ; \mathrm{N}, 12.11, \mathrm{~S}, 6.94$.

2-((1E)-1-\{2-[(2Z)-4-(4-Brom ophenyl)-3-phenyl-2,3-di-hydr 0-1,3-thiazol-2-ylidene]hydrazin-1 -

ylidene\}ethyl) pyridine-1-iumbromide monohydrate (3b). Yellow crystals (ethanol), m.p. $248-250{ }^{\circ} \mathrm{C}(0.47 \mathrm{~g}$, $68 \%), I R(\mathrm{KBr})=3346\left(\mathrm{pyr}^{+} \mathrm{NH}\right), 1614(\mathrm{C}=\mathrm{N}), 1576(\mathrm{~N}-\mathrm{N})$ stretching. ${ }^{1} \mathrm{H}$ NMR $\left(\mathrm{CDCl}_{3}\right) \mathrm{\delta}_{\mathrm{H}}=1.63\left(\mathrm{br}, 2 \mathrm{H}, \mathrm{H}_{2} \mathrm{O}\right), 2.49(\mathrm{~s}$, $\left.3 \mathrm{H}, \mathrm{CH}_{3}\right), 6.41(\mathrm{~s}, 1 \mathrm{H}$, thiazole-CH), 6.97-6.99 (m, 2H, Ar-H), 7.27-7.34 ( $\mathrm{m}, 4 \mathrm{H}, \mathrm{Ar}-\mathrm{H}), 7.36-7.37(\mathrm{~m}, 3 \mathrm{H}, \mathrm{Ar}-\mathrm{H}), 8.23-8.25$ $\left(\mathrm{m}, 4 \mathrm{H}\right.$, pyr-H), $9.10\left(\mathrm{br}, 1 \mathrm{H}, \mathrm{pyr}_{-}^{+} \mathrm{NH}\right) .{ }^{13} \mathrm{C} \mathrm{NMR}\left(\mathrm{CDCl}_{3}\right) \delta_{\mathrm{C}}=14.12\left(\mathrm{CH}_{3}\right), 103.12$ (thiazole-CH), 122.82, 123.56, 128.23, 129.12, 129.92 (Ar-CH), 130.94, 131.27 (pyridine-CH), 128.14, 131.77, 141.03 (Ar-C), 141.63, 141.94 (pyridine-CH), 147.12 ( Pyridine-C), 149.11 (thiazole-C4), 156.38 (acyclic $\mathrm{C}=\mathrm{N}$ ), 168.51 (thiazole-C2). MS. $\mathrm{m} / \mathrm{z}(\%) 466-468\left(\mathrm{M}^{+}-\right.$ $\left.\left(\mathrm{HBr}+\mathrm{H}_{2} \mathrm{O}\right), 15\right), 343$ (16), 315 (28), 135 (43), 105 (81), 77 (100). Anal. Calcd for $\mathrm{C}_{22} \mathrm{H}_{20} \mathrm{Br}_{2} \mathrm{~N}_{4} \mathrm{OS}$ (548.29), C, 48.19; $\mathrm{H}$, 3.68; $\mathrm{Br}, 29.15 ; \mathrm{N}, 10.22 ; \mathrm{S}, 5.85$. Found C, 48.33; H, 3.74; Br, 28.96; N, 10.37; S, 5.69.

(Z)-1-[(2E)-3,4-Diphenyl2,3-dihydro-1,3-thiazol-2-yli-dene]-2-[1-(4-hydr ox yphenyl)ethylide ne]hydrazinium bromide (3c). Yellow crystals (ethanol), $\mathrm{mp} 260-262{ }^{\circ} \mathrm{C}(0.705 \mathrm{~g}, 85 \%)$, IR $(\mathrm{KBr})=3423(\mathrm{OH}), 3332\left(\right.$ hydrazinium $\left.\mathrm{NH}^{+}\right)$, $1616(\mathrm{C}=\mathrm{N}), 1595(\mathrm{Ar} \mathrm{C}=\mathrm{C}), 1564(\mathrm{~N}-\mathrm{N})$ stretching. ${ }^{1} \mathrm{H}$ NMR $\left(\mathrm{CDCl}_{3}\right) \delta_{\mathrm{H}}=2.31\left(\mathrm{~s}, 3 \mathrm{H}, \mathrm{CH}_{3}\right), 6.3(\mathrm{~s}, 1 \mathrm{H}$, thiazole-CH), 6.84$6.99(\mathrm{~m}, 3 \mathrm{H}, \mathrm{Ar}-\mathrm{H}), 7.11-7.18(\mathrm{~m}, 3 \mathrm{H}, \mathrm{Ar}-\mathrm{H}), 7.20-7.29(\mathrm{~m}, 3 \mathrm{H}, \mathrm{Ar}-\mathrm{H}), 7.33-7.55(\mathrm{~m}, 2 \mathrm{H}, \mathrm{Ar}-\mathrm{H}), 7.75-7.77(\mathrm{~m}, 2 \mathrm{H}, \mathrm{Ar}-\mathrm{H})$ $9.51(\mathrm{br}, 1 \mathrm{H}, \mathrm{CH}), 9.20(\mathrm{br}, 1 \mathrm{H}, \mathrm{NH}) .{ }^{13} \mathrm{C}$ NMR $\left(\mathrm{CDCl}_{3}\right) \delta_{\mathrm{C}}=14.15\left(\mathrm{CH}_{3}\right), 101.83$ (thiazole-CH), 122.88, 125.26, 125.54, 126.27, 127.12, 127.88, $128.21(\mathrm{Ar}-\mathrm{CH})$ 124.16, 131.22, 133.12, 142.14 (Ar-C), 148.79 (thiazole-C4), 157.10 (acyclic $\mathrm{C}=\mathrm{N}), 161.05(\mathrm{Ar}-\mathrm{C}-\mathrm{CH}), 168.53$ (thiazole-C2). Ms.m/z (\%) $385\left(\mathrm{M}^{+}-\mathrm{HBr}, 11\right), 370$ (10), 238 (13), 180 (12), 135 (21), 105 
(24), 91 (45), 77 (100). Anal. Calcd for $\mathrm{C}_{23} \mathrm{H}_{20} \mathrm{BrN}_{3} \mathrm{OS}$ (466.39), C, 59.23; H, 4.32; Br, 17.13; N, 9.01; S, 6.88. Found C, 59.37, H, 4.21; $\mathrm{Br}, 16.95 ; \mathrm{N}, 8.87 ; \mathrm{S}, 6.94$.

4-((1E)-1-\{(2Z)-2-[4-(4-brom ophe nyl)-3-phenyl-2,3-dihydr 0-1,3-thiazol-2-ylide ne]hydrazin-1-ylidene\}ethyl)phenol hemihydrate (3d). Yellow crystals (ethanol), mp274-276 ${ }^{\circ} \mathrm{C}(0.454 \mathrm{~g}, 83 \%), \mathrm{IR}(\mathrm{KBr})$

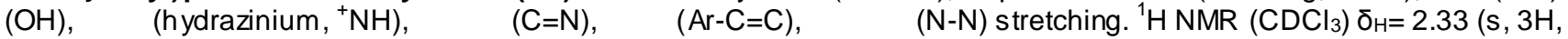
$\left.\mathrm{CH}_{3}\right), 6.21(\mathrm{~s}, 1 \mathrm{H}$, thiazole- $\mathrm{CH}), 6.81-6.94(\mathrm{~m}, 3 \mathrm{H}, \mathrm{Ar}-\mathrm{H}), 7.15-7.21(\mathrm{~m}, 3 \mathrm{H}, \mathrm{Ar}-\mathrm{H}), 7.22-7.30(\mathrm{~m}, 3 \mathrm{H}, \mathrm{Ar}-\mathrm{H}), 7.45-7.61(\mathrm{~m}$, $2 \mathrm{H}, \mathrm{Ar}-\mathrm{H}), 7.79-7.82(\mathrm{~m}, 2 \mathrm{H}, \mathrm{Ar}-\mathrm{H}) 9.63(\mathrm{br}, 1 \mathrm{H}, \mathrm{CH}), 9.28(\mathrm{br}, 1 \mathrm{H}, \mathrm{NH}) .{ }^{13} \mathrm{C} \mathrm{NMR}\left(\mathrm{CDCl}_{3}\right) \delta_{\mathrm{C}}=14.43\left(\mathrm{CH}_{3}\right), 102.15$ (thiazole-CH), 123.11, 124.76, 125.84, 126.32, 127.10, 127.73, 128.31 (Ar-CH) 124.14, 130.55, 132.87, 141.81 (Ar-C), 149.19 (thiazole-C4), $158.12($ acyclic $\mathrm{C}=\mathrm{N}), 160.85(\mathrm{Ar}-\mathrm{C}-\mathrm{CH}), 169.23$ (thiazole-C2).

(1Z,2E)-1-(3,4-Diphenyl-2,3-dihydro-1,3-thiazol-2-yli-dene)-2-(1-p-tolylethylidene)hydrazine (3e). Yellow crystals (ethanol), m.p. $232-234{ }^{\circ} \mathrm{C}(0.31 \mathrm{~g}, 80 \%), \mathrm{IR}(\mathrm{KBr})=3060(\mathrm{Ar}-\mathrm{CH}), 1629(\mathrm{C}=\mathrm{N}), 1594(\mathrm{Ar}-\mathrm{C}=\mathrm{C}), 1567(\mathrm{~N}-\mathrm{N})$ stretching. ${ }^{1} \mathrm{H} \mathrm{NMR}\left(\mathrm{CDCl}_{3}\right), 2.38\left(\mathrm{~s}, 3 \mathrm{H}, \mathrm{CH}_{3}\right), 2.58\left(\mathrm{~S}, 3 \mathrm{H}, \mathrm{CH}_{3}\right), 6.21(\mathrm{~s}, 1 \mathrm{H}$, thiazole- $\mathrm{CH}), 7.08-7.10(\mathrm{~m}, 3 \mathrm{H}, \mathrm{Ar}-\mathrm{H})$, 7.17-7.19 (m, 4H, Ar-H), 7.24-7.26 (m, 4H, Ar-H), 7.43-7.45 (m, 3H, Ar-H); ${ }^{13} \mathrm{C} N M R\left(\mathrm{CDCl}_{3}\right) \delta_{\mathrm{c}}=14.31\left(\mathrm{CH}_{3}\right), 103.14$ (thiazole-CH), 127.08, 128.11, 128.57, 128.77, 128.94, 129.15, 129.56, 129.79 ( Ar-CH), 130.84, 131.42, 132.16 (Ar-C), 142.11 (Ar-C-N), 149.11 (thiazole-C41, 157.62 (acyclic=N), 168.51 (Thiazole-C2). Ms, m/z (\%) $383\left(\mathrm{M}^{+}, 265,368\right.$ (14), 265 (32), 248 (16), 237 (18), 135 (41), 118 (58), 91 (100), 77 (86). Anal. Calcd for $\mathrm{C}_{24} \mathrm{H}_{21} \mathrm{~N}_{3} \mathrm{~S}(383.51), \mathrm{C}, 75.16 ; \mathrm{H}, 5.52$; N, 10.96; S, 8.36. Found C, 74.47; H, 5.41; N, 11.14; S, 8.51.

(2E)-4-(4-Brom ophenyl)-2-\{(2Z)-[1 -(4-methylphe nyl)-ethylidene] hydrazinylidene\}-3-phenyl-2,3-dihydro1,3-thiazole (3f). Yellow crystals (ethanol), m.p. $220-222{ }^{\circ} \mathrm{C}(0.38 \mathrm{~g}, 83 \%), \mathrm{IR}(\mathrm{KBr})=3063(\mathrm{Ar}-\mathrm{CH}), 1612(\mathrm{C}=\mathrm{N}), 1600$ $(\mathrm{Ar}-\mathrm{C}=\mathrm{C}), 1564(\mathrm{~N}-\mathrm{N})$ stretching. ${ }^{1} \mathrm{H} \mathrm{NMR}\left(\mathrm{CDCl}_{3}\right) \delta_{\mathrm{H}}=2.27\left(\mathrm{~s}, 3 \mathrm{H}, \mathrm{CH}_{3}\right), 2.35\left(\mathrm{~s}, 3 \mathrm{H}, \mathrm{CH}_{3}\right), 6.16(\mathrm{~s}, 1 \mathrm{H}$, thiazole-CH$)$, 6.98-7.00 (m, 2H, Ar-CH), 7.73- $7.76(\mathrm{~m}, 3 \mathrm{H}, \mathrm{Ar}-\mathrm{H}) .{ }^{13} \mathrm{C}$ NMR $\left(\mathrm{CDCl}_{3}\right) \delta_{\mathrm{c}}=14.84\left(\mathrm{CH}_{3}\right), 21.30\left(\mathrm{CH}_{3}\right), 102.29($ thiazole $-\mathrm{CH})$, 126.27, 127.48, 128.25, 128.75, 128.87, 129.51, $129.73(\mathrm{Ar}-\mathrm{H}), 124.63,131.46,132.16,132.36,141.83$ (Ar-C), 148.86 (thiazole-CH), 157.52 (acyclic $\mathrm{C}=\mathrm{N}), 168.52$ ( thiazole-C2). MS, m/z (\%) 461/463 (M+, 21), $381(16), 135(20), 118(93), 41$ (81), 77 (100). Anal. Calod for $\mathrm{C}_{24} \mathrm{H}_{20} \mathrm{BrN}_{3} \mathrm{OS}$ (462.40) C, 62.34; H, 4.36; Br, 17.28; N, 9.09; S, 6.93. Found C, 62.19; H, $4.45 ; \mathrm{Br}, 17.41 ; \mathrm{N}, 8.92 ; \mathrm{S}, 7.11$.

(E)-2-\{(E)-[1-(4-Chlor ophenyl)ethylidene]hydrazono\}-3,4-diphenyl-2,3-dihydrothiazole (3g). Yellow crystals (ethanol), $\mathrm{mp} .176-178{ }^{\circ} \mathrm{C} \quad(0.36 \mathrm{~g}, 89 \%), \mathrm{IR}(\mathrm{KBr})=3048(\mathrm{Ar}-\mathrm{CH}), 1603(\mathrm{C}=\mathrm{N}), 1590(\mathrm{Ar}-\mathrm{C}=\mathrm{C}), 1569(\mathrm{~N}-\mathrm{N})$ stretching. ${ }^{1} \mathrm{H} \mathrm{NMR}\left(\mathrm{CDCl}_{3}\right) \delta_{\mathrm{H}}=2.30\left(\mathrm{~s}, 3 \mathrm{H}, \mathrm{CH}_{3}\right), 6.17(\mathrm{~s}, 1 \mathrm{H}$, thiazol-CH), 7.11-7.13 (m, 2H, Ar- $\mathrm{H}), 7.19-7.21(\mathrm{~m}, 4 \mathrm{H}, \mathrm{Ar}-\mathrm{H}), 7.24-$ $7.26(\mathrm{~m}, 4 \mathrm{H}, \mathrm{Ar}-\mathrm{H}), 7.29-7.32(\mathrm{~m}, 2 \mathrm{H}, \mathrm{Ar}-\mathrm{H}), 7.78-7.80(\mathrm{~m}, 2 \mathrm{H}, \mathrm{Ar}-\mathrm{H}) .{ }^{13} \mathrm{C}$ NMR $\left(\mathrm{CDCl}_{3}\right) \delta_{\mathrm{C}}=14.68\left(\mathrm{CH}_{3}\right), 101.72$ (thiadiazole-CH), 127.45, 127.59, 128.26, 128.34, 128.39, 128.41, 128.68, 128.83 (Ar-CH), 131.34, 131.89, 132.42, 141.66 (Ar-C), 149.12 (thiazole-C4), 157.68 (acyclic C=N), 169.67 (thiazole-C2). Ms, m/z (\%) 403/405 (M+, 26$), 388(14)$, 367 (15), 260 (23), 232 (19), 167 (62), 135 (49), 91 (100), 77 (92). Anal. Calcd for $\mathrm{C}_{23} \mathrm{H}_{18} \mathrm{CIN} \mathrm{N}_{3} \mathrm{~S}(403.93) \mathrm{C}, 68.39, \mathrm{H}, 4.49$, $\mathrm{Cl}, 8.78 ; \mathrm{S}, 7.94$. Found C, 68.53; $\mathrm{H}, 4.37 ; \mathrm{Cl}, 8.62 ; \mathrm{S}, 8.12$.

(E)-4-(4-Bromophenyl)-2-\{(E)-[1-(4-Chlorophenyl)-ethylidene]hydrazono\}-3-phenyl-2,3-dihydrothiazol

(3h). Yellow crystal (ethanol), m.p. $186-188{ }^{\circ} \mathrm{C}(0.392 \mathrm{~g}, 81 \%), \mathrm{IR}(\mathrm{KBr})=3076(\mathrm{Ar}-\mathrm{CH}), 1605(\mathrm{C}=\mathrm{N}), 1585(\mathrm{Ar}-\mathrm{C}=\mathrm{C})$, $1562(\mathrm{~N}-\mathrm{N})$ stretching. ${ }^{1} \mathrm{H}$ NMR $\left(\mathrm{CDCl}_{3}\right) \delta_{\mathrm{H}}=2.29\left(\mathrm{~S}, 3 \mathrm{H}, \mathrm{CH}_{3}\right), 6.19(\mathrm{~S}, 1 \mathrm{H}$, thiazole-CH), 6.97-6.99 $(\mathrm{m}, 2 \mathrm{H}, \mathrm{Ar}-\mathrm{H}), 7.25-$ $7.29(\mathrm{~m}, 4 \mathrm{H}, \mathrm{Ar}-\mathrm{H}), 7.31-7.35(\mathrm{~m}, 4 \mathrm{H}, \mathrm{Ar}-\mathrm{H}), 7.77-7.81(\mathrm{~m}, 3 \mathrm{H}, \mathrm{Ar}-\mathrm{H}) .{ }^{13} \mathrm{C} \mathrm{NMR}\left(\mathrm{CDCl}_{3}\right) \delta_{\mathrm{C}}=14.59\left(\mathrm{CH}_{3}\right), 102.50($ thiazole$\mathrm{CH}), 127.50,127.55,128.22,128.29,128.76,129.12,129.52(\mathrm{Ar}-\mathrm{CH}), 130.13,131.47,131.76,132.14,141.26(\mathrm{Ar}-\mathrm{C})$, 149.11 (thiazole-C4), 155.97 (acyclic $\mathrm{C}=\mathrm{N}$ ), 169.26 (thiazole $\mathrm{C} 2$ ) Ms, $\mathrm{m} / \mathrm{z}(\%)$ 481/485 $\left(\mathrm{M}^{+}, 14\right), 445 / 447(26), 430(11)$, 401 (25), 342 (11), 167 (10), 139 (32), 135 (41), 91 (82), 77 (100). Anal. Calcd for $\mathrm{C}_{23} \mathrm{H}_{17} \mathrm{BrClN}_{3} \mathrm{~S}$ (482.82) C, 57.21; H, $3.55 ; \mathrm{Br}, 16.55 ; \mathrm{Cl}, 7.34 ; \mathrm{N}, 8.70 ; \mathrm{S}, 6.64$. Found, C, 57.07; H, 3.62; Br, 16.69; Cl, 7.28; N, 8.56; S, 6.76.

\section{Anti-bacterial Activity}

The synthesized compounds 3a-h were dissolved in DMSO. In order to ensure that the solvent had no effect on bacterial growth or enzymatic activity, negative control tests were performed using DMSO at the same concentrations.

The inhibitory effect of tested compounds $\mathbf{3 a - h}$ on the in vitro growth of four different types of bacteria Bacillus cereus and Micrococcus lutues as gram positive bacteria (+ve) and Psedomonas aureginosa and Serratia marcescens as gram negative bacteria (-ve) was evaluated using agar diffusion method (cup and plate method) [45-48]. All plates were incubated at $37 \pm 0.5^{\circ} \mathrm{C}$ for $24 \mathrm{~h}$. The inhibition zone of active compounds was measured in $\mathrm{cm}$ scale. The results shown in table 1 revealed that compounds $\mathbf{3 h}$ and $\mathbf{3} \mathbf{f}$ showed the highest inhibitory effect against the two types of bacteria at the three used concentrations, while compounds $\mathbf{3 d}$ and $\mathbf{3 e}$, showed moderate activity compared to the standard Moxifloxacin as a broad spectrum antibiotic. Compound $3 \mathrm{~h}$ exhibited the highest inhibitory effect against the gram positive Bacillus cereus at high concentration while $\mathbf{3} \mathbf{c}$ showed high inhibition zone against the gram positive bacteria Micrococcus lutues at low concentration. On the other hand, compounds $\mathbf{3 a - c}, \mathbf{3 e}$ and $\mathbf{3 g}$ showed no bactericidal activity against the gram negative bacterial Psedomonas aureginosa (table 1). 
Table 1: Anti-bacterial activity of compounds 3a-h

\begin{tabular}{|c|c|c|c|c|c|c|c|c|c|c|c|}
\hline \multicolumn{2}{|c|}{ Bacteria } & & $3 \mathbf{a}$ & $3 \mathbf{b}$ & $3 c$ & $3 d$ & $3 e$ & $3 f$ & $3 \mathrm{~g}$ & 3h & Moxifl- \\
\hline \multirow{6}{*}{$\begin{array}{l}\text { Gm- } \\
\text { Bacteria }\end{array}$} & \multirow{3}{*}{$\begin{array}{c}\text { Pseudomonas } \\
\text { aeruginosa }\end{array}$} & 10000 & $=$ & - & $=$ & 0,4 & $=$ & - & $=$ & 0.8 & 1.4 \\
\hline & & 30000 & - & - & - & 0.7 & - & 0.2 & - & 1.0 & 1.5 \\
\hline & & 50000 & - & - & - & 0.7 & - & 0.4 & - & 1.1 & 1.4 \\
\hline & \multirow{3}{*}{$\begin{array}{c}\text { Serratia } \\
\text { marcescens }\end{array}$} & 10000 & - & 0.1 & - & 0.3 & 1.1 & 1.3 & 0.5 & 1.5 & 1.5 \\
\hline & & 30000 & - & 0.2 & - & 0.6 & 1.4 & 1.4 & 0.7 & 1.7 & 1.6 \\
\hline & & 50000 & - & 0.2 & - & 0.6 & 1.7 & 1.6 & 0.9 & 1.8 & 1.6 \\
\hline \multirow{6}{*}{$\underset{\text { Bacteria }}{\mathrm{Gm+}}$} & \multirow{3}{*}{$\begin{array}{l}\text { Bacillus } \\
\text { cereus }\end{array}$} & 10000 & - & 0.2 & 1.0 & 1.1 & 1.6 & 1.2 & 1.0 & 1.6 & 1.6 \\
\hline & & 30000 & - & 0.2 & 1.2 & 1.5 & 1.5 & 1.7 & 0.8 & 1.8 & 1.8 \\
\hline & & 50000 & - & 0.4 & 1.4 & 1.4 & 1.4 & 1.7 & 0.6 & 1.8 & 1.8 \\
\hline & \multirow{3}{*}{$\begin{array}{c}\text { Micrococcus } \\
\text { lutues }\end{array}$} & 10000 & 0.2 & 0.5 & 1.5 & 1.0 & 1.3 & 1.1 & 0.1 & 0.9 & 0.8 \\
\hline & & 30000 & 0.3 & 0.6 & 1.3 & 1.3 & 1.2 & 1.0 & 0.1 & 1.1 & 1.0 \\
\hline & & 50000 & 0.4 & 0.8 & 1.1 & 1.2 & 1.1 & 0.9 & 0.1 & 1.1 & 1.1 \\
\hline
\end{tabular}

\section{ACKNOWLEDGMENTS}

Authors would like to express their deep thanks to Manchester Metropolitan University, Minia University and Sohag University for supporting this sudy.

\section{REFERENCES}

[1] Wang, L. F., Li, Y. Z., Li, Q. X., Xu, Z. D. and Qu, D. M. 2001, Trans. Met. Chem., 2001, 26, 307-310.

[2] Yadav, L. D. S.; Singh, S.; Indian J. Chem., 40B, 2001, 440-442.

[3] Cukurovali, A.; Yilmaz, I.; Gur, S.; Kazaz, C. Eur. J. Med. Chem., 2006, 41, 201-207.

[4] Gruttadauria, M.; Buccheri, F.; Cusmano, G., Meo, P. L.; Noto, R.; Werber, G., J. Heterocycl. Chem., 1993, 30, 765770

[5] Gautam, D.; Gautam, P.; Chaudhary, R. P., Heterocycl. Commun., 2011, 17, 147-150.

[6] Saydam, S.; Yilmaz, E., Spectrochim. Acta, 2006, 63A., 506-510

[7] Luo, L.; Meng, L.; Sun, Q.; Ge, Z.; Li, R. Tetrahedron Lett., 2014, 55, 259-263.

[8] De, S.; Adhikari, S.; Tilak-Jain, J.; Menon, V. P.; Devasagayam, T. P. A., Chemico-Bilogical Interactions, 2008, 173, 215-223.

[9] Holla, B. S.; Malini, K. V.; Rao, B. S.; Sarojini, B. K.; Kumari, N. S., Eur. J. Med. Chem., 2003, 38, $313-318$.

[10] Verma, A.; Saraf, S. K., Eur. J. Med. Chem., 2008, 43, 897-905.

[11] Aridoss, G., Amirthaganesan, S.; Kim, M. S., Kim, J. T,; Jeong, Y. T., Eur. J. Med. Chem., 2009, 44, 4199-4210

[12] Sup, R. C.; Sup, R. Y.; Bang, C. W., Korean J. Med. Chem., 1995, 5, 72-75

[13] Vukovic, N.; Sukdolak, S.; Solujic, S.; Milosevic, T., Arch. Pharm. (Weinheim), 2008, 341, 491-496.

[14] Röver, S.; Cesura, M. A.; Huguenin, P.; Kettler, R.; Szente, A., J. Med. Chem., 1997, 40, 4378-4385.

[15] Franklin, P. X.; Pillai, A. D.; Rathod, P. D.; Yerande, S.; Nivsarkar, M.; Padh, H.; Vasu, K. K.; Sudarsanam, V., Eur. J. Med. Chem., 2008, 43, 129-134

[16] Geronikaki.; A. Hadjipavlou-litina, D.; Amourgianou, M., II Farmaco, 2003, 58, 489-495.

[17] Amin, K. M.; Rahman, A. D. E.; Al-Eryani; Y. A., Bioorg. Med. Chem., 2008, 16, 5377-5388

[18] Raumagrass, R.; Weiwad, M.; Erdmann, F.; Liu, J. O.; Wunderlick, D.; Grabley, S.; Fischer, G., J. Biol. Chem., 2001, 276, 47914-47921.

[19] Rao, A., Balzarini, J.; Carbone, A.; Chimirri, A., De Clercq, E.; Monforte, A M.; Monforte, P., Pannecouque, C.; Zappalà, M., II Farmaco, 2004, 59, 33-39.

[20] Rao, A.; Carbone, A.; Chimirri, A.; De Clercq, E.; Mouforte, A. M.; Manforte, P.; Christophe Pannecouque., C.; Zappala, M., II Farmaco, 2002, 57, 747-751.

[21] Li, J.; Du, J.; Xia, L.; Liu, H.; Yao, X.; Liu, M., Analytica Chemica Acta, 2009, 631, 29 -39.

[22] Gorczynski.; M. J.; Leal, R. M.; Mooberry, S. L,; Bushweller, J. H.; Brown, M. L., Bioorg. Med, Chem., 2004, 12, 1029-1036. 
[23] Hang, P.C.; Honek, J. F., Bioorg. Med, Chem. Lett., 2005, 15, 1471-1474.

[24] Zhao, R., Gove, S.; Sundeen, J. E.; Chen, B.-C., Tetrahedron lett., 2001, 42, 2101-2102

[25] Wiley, R. H.; England, D. C.; Behr, L. C. Organic Reactions John Wiley, 1951, 6, 367.

[26] Thomae, D.; Perspicace, E.; Xu, Z.; Henryon, D.; Schneider, S.; Hesse, S.; Kirsch, G.; Seck, P., Tetrahedron, 2009, $65,2982-2988$

[27] Dunst, C.; Knochel, P., J. Org. Chem., 2011, 76, 6972-6978.

[28] Li, Z.; Ma, L.; Tang, C.; Xu, J.; Wu, X.; Yao, H., Tetrahedron Lett. 2011, 52, 5643-5647.

[29] Hassan, A. A.; Ibrahim, Y. R.; El-Sheref, E. M.; Abdel-Aziz, M.; Bräse, S.; Nieger, M., Arch. Pharm. Life Sci., 2013, 346, 562-570.

[30] Dhanuskodi.; S.; Manivannan, S.; Kirschbaum, K., Spectrochim Acta, 2006, 64A, 504-511.

[31] Dhanuskodi.; S.; Manivannan, S.; Philip, J., Spectrochim Acta, 2008, 69A, 1207-1212.

[32] Azzouz, R.; Fruit, C., Bischoff, L., Marsais, F., J. Org. Chem., 2008, 73, 1154-1157.

[33] Manivannan, S.; Tiwari, S. K.; Dhanuskodi, S., Solid State Commun., 2004, 132, $123-127$.

[34] Seethalakshim.; T.; Kaliannan, P.; Venkatesan, P.; Froncezk, F. R.; Thamotharan, S., Acta Cryst., 2006, E62, 02353 02355 and referenes therein

[35] Akkurt. M.; Mague, J. T.; Mohamed, S. K.; Hassan, A. A.; Albayati, M. R., Acta Cryst., 2014, E70, 0478-0479.

[36] Mague, J. T.; Mohamed, S. K.; Akkurt, M.; Abd El-Aziz, A. T.; Albayati, M. R, Acta Cryst, 2014, E70, 0328-0329

[37] Mague, J. T.; Mohamed, S. K.; Akkurt, M.; Hassan, A. A.; Albayati, M. R, Acta Cryst,,2014, E70, 0647 -0648

[38] Mague, J. T., Akkurt, M., Mohamed, S. K., Hassan, A. A. and Albayati, M. R. Acta Cryst,,2014, E70, $01124-01125$.

[39] Mohamed, S. K.; Mague, J. T.; Akkurt, M.; Hassan, A. A.; Albayati, M. R., Acta Cryst, 2013, E69, $01324-01324$

[40] Mohamed, S. K., Akkurt, M.; Mague, J. T.; Hassan, A. A.; Albayati, M. R., Acta Cryst, 2013, E69, 01563-01564

[41] Sheribani.; H.; Mosslemin, M. H,; Behzadi.; S., Islami, M. R.; Forought, H., Saidi, K., Arki voc, 2005, xv, 88-96.

[42] Mohamed, H. M.; Abdel-Wahab, A-H. F.; Ahmed, K. A.; El-Agrody, A. M.; Bedair, A. H.; Eid, F. A.; Khafagy, M. M., Molecules, 2012, 17, 971-988.

[43] Nobuta, T.; Hirashima, S.; Tada, N.; Miura, T.; Itoh, A., Synlett, 2010, 2335-2339.

[44] Salama, T. A.; Novàk, Z., Tetrahedron lett., 2011, 52, 4026-4029.

[45] Jawetz, E.; Melnick, J. L.; Adelberg, E. A. Review of Medical Microbiology, Lang Medical Pulication, Los Altos, Califomia, 1974.

[46] Grayer, J. R.; Harbone, J. B. Phytochemistry 1994, 37 19-42.

[47] Muanza, D. N.; Kim, B. W.; Euler, K. L.; Williams, L. Pharm. Biol. 1994, 32, 337-345.

[48] Iroi, O. N.; Moo-Young, M.; Anderson, W. A. ; Pharm. Biol. 1996, 34, 87-90.

\section{Author' biography with Photo}

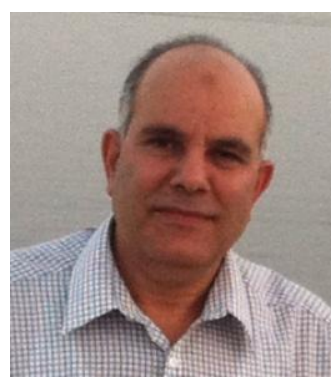

\section{Dr. Shaaban K. Mohamed}

Chemistry Department, Faculty of Science, Minia University, Egypt. Visiting scientists in various universities in United Kingdom and Germany. On 2011appointed as a visiting professor of Organic and Bio-Organic Chemistry based developing research at Manchester Metropolitan University, Manchester, England. Awarded the knowledge extchange award for 2013 from same university and act as Editor and peer reviewer in many scientific joumals. 